\title{
Prevalence, Causes, and Factors Associated with Visual Impairment in a Chinese Elderly Population: The Rugao Longevity and Aging Study
}

\author{
Hangqi Shen, ${ }^{1-6, *}$ Hui Zhang, (iD ${ }^{7,8, *}$ \\ Wei Gong, ${ }^{1-6}$ Tianwei Qian, ${ }^{1-6}$ \\ Tianyu Cheng, ${ }^{1-6}$ Li Jin, ${ }^{7,8}$ \\ Xiaofeng Wang, ${ }^{7-10}$ Dawei Luo, ${ }^{1-6}$ \\ Xun $\mathrm{Xu}^{\mathrm{I}-6}$ \\ 'Department of Ophthalmology, Shanghai \\ General Hospital, Shanghai Jiao Tong \\ University School of Medicine, Shanghai, \\ People's Republic of China; ${ }^{2}$ Shanghai Key \\ Laboratory of Ocular Fundus Diseases, \\ Shanghai, People's Republic of China; \\ ${ }^{3}$ Shanghai Engineering Center for Visual \\ Science and Photomedicine, Shanghai, \\ People's Republic of China; ${ }^{4}$ Shanghai \\ Engineering Center for Precise Diagnosis \\ and Treatment of Eye Diseases, Shanghai, \\ People's Republic of China; ${ }^{5}$ National \\ Clinical Research Center for Eye \\ Diseases, Shanghai, People's Republic of \\ China; ${ }^{6}$ Key Laboratory of Arrhythmias \\ of the Ministry of Education of China, \\ Tongji University School of Medicine, \\ Shanghai, People's Republic of China; \\ ${ }^{7}$ Ministry of Education Key Laboratory of \\ Contemporary Anthropology, School of Life \\ Sciences, Fudan University, Shanghai, People's \\ Republic of China; ${ }^{8}$ Human Phenome \\ Institute, Fudan University, Shanghai, \\ People's Republic of China; 'Shanghai Key \\ Laboratory of Clinical Geriatric Medicine and \\ Huadong Hospital Clinical Research Center \\ for Geriatric Medicine, Shanghai, People's \\ Republic of China; ${ }^{10} \mathrm{National}$ Clinical \\ Research Center for Aging and Medicine, \\ Huashan Hospital, Fudan University, \\ Shanghai, People's Republic of China
}

*These authors contributed equally to this work

Correspondence: Dawei Luo; Xun Xu Department of Ophthalmology, Shanghai General Hospital, Shanghai Jiao Tong University School of Medicine, Shanghai, People's Republic of China

Emaildr-davie@yeah.net; drxuxun@sjtu. edu.cn
Purpose: This study aimed to explore the current prevalence, causes, and factors associated with visual impairment in a Chinese elderly population.

Methods: A random sample of 2164 candidates aged $\geq 70$ years was selected. Among them, 1914 participants (response rate: 88.4\%) underwent comprehensive eye examinations. The prevalence and causes of visual impairment were estimated, and the associated factors were identified.

Results: The standardized prevalence of mild visual impairment $(<6 / 12$ to $\geq 6 / 18)$, moderate to severe visual impairment (MSVI) $(<6 / 18$ to $\geq 3 / 60)$, and blindness $(<3 / 60)$ in the better eye were $20.5 \%, 25.8 \%$, and $3.4 \%$, respectively. The leading cause of visual impairment was cataract $(49.7 \%)$, followed by uncorrected refractive error $(26.5 \%)$, myopic maculopathy $(5.8 \%)$, and posterior capsular opacification (5.5\%). Optical coherence tomography revealed that vitreoretinal interface abnormalities were the third most common cause of monocular mild visual impairment (2.2\%) and MSVI (4.4\%) in the elderly population. A large number of patients with visual impairment $(81.7 \%)$ were amenable to undergo the interventions. Visual impairment was associated with advanced age (odds ratio [OR], 1.09 per year; $p<0.001)$, female sex (OR, 1.59; $p=0.003$ ), self-reported visual impairment (OR, 1.91; $p<0.001$ ), cognitive impairment (OR, 1.40, $p=0.005$ ), and high educational level (OR, 0.75 ; primary, $p=0.045$; and OR, 0.53 , secondary or higher; $p<0.001$ ).

Conclusion: Visual impairment was common in the Chinese elderly population and a severe health and social problem. Practicable policies are urgently needed to popularize eye health knowledge and promote treatments for visual impairment in elderly people in rural China.

Keywords: visual impairment, blindness, elderly, aging

\section{Plain Language Summary}

Vision impairment is common in later life. It may severely impact the quality of life and subjective well-being, and increase the risk of disability and depression in older age. However, little attention has been paid to investigating this phenomenon occurring among older adults.

Our findings show that nearly half of the elderly individuals aged $\geq 70$ years living in rural China had visual impairment, which was predominantly treatable Cataract and uncorrected refractive error remain the leading causes of vision loss, highlighting the significant challenges in popularizing cataract surgery and the use of spectacles for the elderly individuals. In particular, optical coherence tomography revealed that vitreoretinal interface abnormality was a frequent cause of visual impairment in the elderly population. Advanced age, female sex, self-reported visual impairment, cognitive impairment, and low education level were associated with a higher prevalence of visual impairment. 
Given the rapid growth of the elderly population, practicable policies, such as raising awareness of eye healthcare and increasing cataract surgery coverage, are urgently needed to promote visual impairment rehabilitation services in the Chinese elderly population.

\section{Introduction}

Visual impairment among adults remains an important public health problem worldwide. ${ }^{1}$ In the elderly population, visual impairment severely impacts the quality of life and subjective well-being, and increases the risk of disability and depression. $^{2-4}$ China has the world's largest population and is facing a rapid growth in the elderly population. By 2050 , the number of elderly individuals is projected to reach approximately one quarter of the national population. ${ }^{5}$ Visual impairment substantially aggravates the social and medical burdens led by societal aging, which are much more serious in rural areas than that in urban areas due to the rural-urban migration of young people. ${ }^{6}$

Unfortunately, to date, the number of population-based epidemiological studies on visual impairment in elderly people in China is limited. Several studies have reported the prevalence of visual impairment in patients aged 40 years and older, but little attention has been paid to assessing people aged $\geq 70$ years. ${ }^{7-10}$ Moreover, the most recent survey on visual impairment was conducted before 2014. Therefore, an updated epidemiologic study is warranted to provide a more precise assessment of the visual health status in the rapidly growing elderly population. This study aimed to determine the current prevalence, causes, and associated factors of visual impairment in a Chinese elderly population.

\section{Materials and Methods}

\section{Study Population}

The Rugao Longevity and Aging Study (RuLAS) is an ongoing, dynamic, population-based, observational cohort study conducted in Rugao, a typical medium-sized county in Jiangsu Province, China. The cohort study aimed to investigate the prevalence, trajectories, and risk factors of various age-related phenotypes in a Chinese elderly population. The study protocol of RuLAS has been reported before. $^{11}$ In brief, 1788 elderly individuals aged 70-84 years from 31 rural communities in Jiang'an township, one of the 14 townships in Rugao, were randomly recruited using the 5-year age and gender strata in November 2014 (Wave 1). Follow-up surveys were conducted 1.5 years later (April-June 2016, Wave 2), 3 years later (November- December 2017, Wave 3), and 5 years later (December 2019 - January 2020, Wave 4). A flow diagram of the aging arm of the RuLAS is shown in Supplementary Figure.

We performed ophthalmic examinations on 2200 participants (aged 60-97) in Wave 4. Among them, 2164 participants of the fourth-wave cohort aged $\geq 70$ years were enrolled in the current study. This study was approved by the Human Ethics Committee of the School of Life Sciences of Fudan University and the Ethics Committee of Shanghai General Hospital, Shanghai Jiao Tong University School of Medicine. Informed consent was obtained from all participants according to the Declaration of Helsinki.

\section{Clinical and Ophthalmic Examinations}

All recruited participants were invited to the local clinics. Fasting blood samples were collected for biochemical tests (eg, complete blood count and hemoglobin A1c). Physical examinations (eg, grip strength test and blood pressure measurement) were performed by trained physicians from Rugao People's Hospital. Body mass index (BMI, weight in kilograms divided by height in meters squared) was categorized into four groups: underweight $(<18.0)$, normal (18.0-23.9), overweight (24.0-27.9), and obese ( $\geq 28.0)$. This classification of BMI is used as the gold standard in the Chinese population. ${ }^{12}$ A structured face-to-face questionnaire (eg, sociodemographic characteristics, lifestyles, and cognitive function) was completed by each participant. All interviews were tape recorded, and 5\% of the recordings were randomly evaluated for quality of interviews. Cognitive assessment was performed using the Mini-Mental State Examination (MMSE), which is the most widely used tool for screening cognitive function in older adults. ${ }^{13}$ Cognitive impairment was defined as MMSE scores of $\leq 6$ for illiterate, $\leq 19$ for individuals with $1-6$ years of education, and $\leq 23$ for individuals with 7 or more years of education.

Ophthalmic examination was performed using a standardized protocol. Presenting visual acuity (PVA, wearing current optical correction if any) was measured using a retro-illuminated logarithm of the minimum angle of resolution $\mathrm{E}$ chart at a distance of $4 \mathrm{~m},{ }^{14}$ and the eyes were measured separately. Visual acuity test was used to determine the smallest line read with one or no errors. Participants failing to read the top line at $1 \mathrm{~m}$ were tested for finger counting, hand movement, or light perception. If PVA was less than 6/12 in either eye, the best-corrected visual acuity (BCVA) was measured using the pinhole test. The intraocular pressure was measured using the Icare rebound tonometer (ic100, TAO11, Helsinki, Finland). 
Anterior and posterior segment assessments were performed by an experienced ophthalmologist (Dr. HS) using a slit lamp (YZ3, 66 Vision Tech Co., Ltd., Suzhou, China) and direct ophthalmoscopy (YZ11D, 66 Vision Tech Co., Ltd., Suzhou, China) without pupillary dilation. For each eye, fundus photographs $\left(45^{\circ}\right)$ showing the macular and optic discs were obtained using a nonmydriatic hand-held fundus camera (MicroClear Medical Instruments Co., Suzhou, China) in a darkened room to allow for physiological mydriasis. Spectral-domain optical coherence tomography (OCT) (HS100, Canon Inc., Tokyo, Japan) images were obtained using a macular 3D scan within a $10 \times 10 \mathrm{~mm}$ area.

\section{Definitions of Visual Impairment}

Bilateral visual impairment was defined as a PVA of $<6 / 12$ in the better eye, according to the World Health Organization (WHO) criteria. ${ }^{1}$ Visual impairment was subdivided into mild visual impairment (PVA $<6 / 12$ and $\geq 6$ / 18), moderate to severe visual impairment (MSVI) (PVA $<6 / 18$ and $\geq 3 / 60$ ), and blindness (PVA $<3 / 60$ ). The United States criteria was also used, which defined visual impairment as PVA values of $<6 / 12$ and $\geq 6 / 60$ in the better eye and blindness as a PVA value of $<6 / 60$ in the better eye. ${ }^{15}$

Unilateral vision impairment was also defined as follows: ${ }^{16}$ (1) mild (PVA $<6 / 12$ in the worse eye and $\geq 6 / 12$ in the better eye), (2) moderate to severe (PVA $<6 / 18$ in the worse eye and $\geq 6 / 18$ in the better eye), and (3) blindness (PVA $<3 / 60$ in the worse eye and $\geq 3 / 60$ in the better eye).

\section{Causes of Visual Impairment}

Eye conditions were determined by an ophthalmologist (Dr. HS). Two experienced ophthalmologists (Drs. HS and WG) determined the principal cause of visual impairment based on clinical history and examination records. If a primary diagnosis was not definite during the initial assessment, the final adjudication was made by a senior ophthalmologist (Dr. TQ). The principal causes were determined according to the following itemized list: ocular atrophy or prosthetic eye, amblyopia, pterygium, corneal opacity, uncorrected refractive error, cataract, posterior capsular opacification, glaucoma, other optic atrophy, uveitis, age-related macular degeneration, myopic maculopathy, diabetic retinopathy, retinal detachment, vitreoretinal interface abnormality, other posterior segment eye disease, and undetermined disease. In eyes with two or more pathological alterations that might lead to visual impairment, the cause with the greatest impact on visual acuity was designated as the principal cause.
At the individual level, if the causes of visual impairment both eyes were different, the cause that could be more easily treated was designated as the principal cause. ${ }^{17}$ For instance, if uncorrected refractive error was the cause of visual impairment in one eye and cataract in the other eye, the principle cause of visual impairment was considered uncorrected refractive error because the disease is easier to manage compared with cataract surgery.

The presence of cataract was determined based on the Lens Opacities Classification System III. ${ }^{18}$ Cataract was considered as the main cause of visual impairment if the fundus details were obscured due to significant lens opacity without evidence of other ocular abnormalities. Visual impairment was attributed to uncorrected refractive error when the PVA in the better eye was initially $<6 / 12$ and improved to $\geq 6 / 12$ after performing a refractive correction. ${ }^{19}$ Myopic maculopathy was defined according to a simplified meta-analysis of the pathologic myopia classification system. ${ }^{20}$ The prevalence of vitreoretinal interface abnormality was determined by fundus photography and OCT, as defined by the International Vitreomacular Traction Study Group, including the epiretinal membranes, vitreomacular traction, full thickness macular hole, and lamellar macular hole. ${ }^{21}$ We assumed that visual impairment caused by cataract, uncorrected refractive error, and posterior capsular opacification were amenable to interventions such as cataract surgery, spectacle correction, and Nd:YAG laser capsulotomy. ${ }^{17}$

\section{Statistical Analysis}

The categorical data were analyzed using the chi-square test, while continuous data were analyzed using the $t$-test (after Bonferroni correction). The age- and genderstandardized prevalence of visual impairment was estimated based on the latest China National Census (2010), with its $95 \%$ confidence intervals (CIs). Univariate logistic regression analyses were used to investigate the relationships between demographic factors and bilateral visual impairment, and variables with a $p$ value of $<0.05$ were included in the multivariate models. A $p$ value (two tailed) less than 0.05 was considered significant.

\section{Results}

\section{Study Population}

A flow diagram of study participants is shown in Figure 1. Among the total cohort of 2164 individuals aged $\geq 70$ years, 1914 (response rate, 88.4\%) eligible participants 


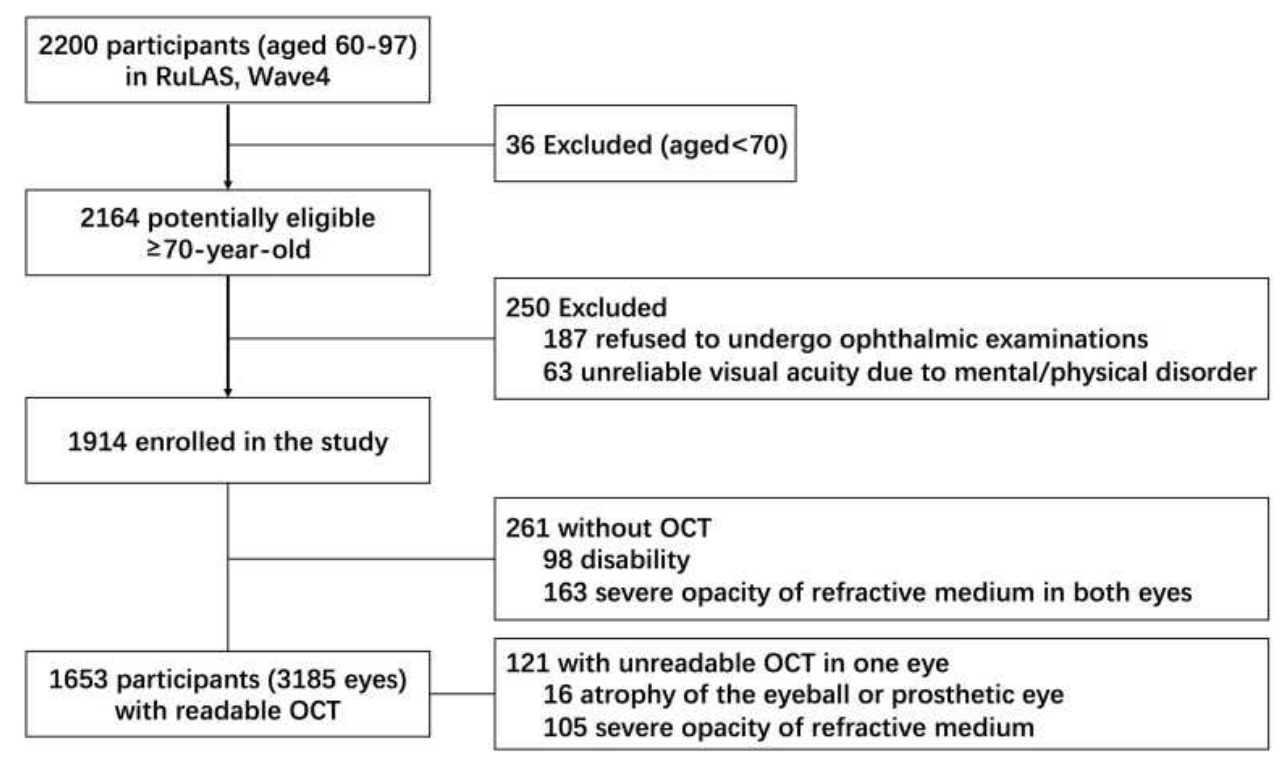

Figure I Flow diagram of study participants.

Abbreviations: RuLAS, Rugao Longevity and Aging Study; OCT, optical coherence tomography.

completed the eye examinations. The mean age of the participants was $79.1 \pm 4.6$ years (median [range], 79 [70-97] years), which was younger than the mean age of the non-participants $(80.1 \pm 4.9, p=0.002)$ (Table 1). Approximately $54.0 \%$ of the participants were women. Among the 1914 participants constituting the analysis cohort, readable OCT images were available in 1653 (76.4\%) participants (3185 eyes). No significant difference was observed in the mean age $(p=0.677)$ or the prevalence of visual impairment $(p=0.830)$ between patients with and without OCT scan.

\section{Prevalence of Visual Impairment}

Based on the WHO criteria, the age- and gender-standardized prevalence of bilateral presenting visual impairment was $48.9 \%$ (95\% CI, 46.7-51.2\%), which included mild visual impairment in $20.5 \%$ (95\% CI, $18.7-22.3 \%)$, MSVI in $25.8 \%$ (95\% CI, 23.9-27.8\%), and blindness in 3.4\% (95\% CI, 2.6-4.2\%). The age- and gender-standardized prevalence of bilateral bestcorrected visual impairment was 33.1\% (95\% CI, 31.0-35.2\%), which included mild visual impairment in $12.4 \%$ (95\% CI, 10.9-13.8\%), MSVI 17.7\% (95\% CI, 16.0-19.4\%), and blindness in 3.0\% (95\% CI, 2.2-3.8\%) (Table 2).

Based on the United States criteria, the age- and genderstandardized prevalence of bilateral presenting visual impairment and blindness were $43.5 \%$ (95\% CI, 41.3-45.7\%) and $5.5 \%$ (95\% CI, 4.5-6.5\%), respectively. The age- and gender-standardized prevalence of bilateral best-corrected visual impairment and blindness were $28.2 \% \quad(95 \% \quad$ CI,
$26.1-30.2 \%)$ and $4.9 \%(95 \%$ CI, $4.3-5.9 \%)$, respectively (Supplementary Table 1).

\section{Causes of Visual Impairment}

Cataract (49.7\%) was the major cause of bilateral visual impairment, followed by uncorrected refractive error (26.5\%) and myopic maculopathy (5.8\%) (Table 3). Uncorrected refractive error was the most frequent cause of mild bilateral visual impairment. The main causes of blindness were cataracts (28.0\%), myopic maculopathy (20.7\%), and corneal opacity $(12.2 \%)$.

The leading causes of monocular visual impairment were cataract $(60.1 \%)$, uncorrected refractive error (14.4\%), myopic maculopathy (3.8\%), and vitreoretinal interface abnormalities (3.5\%) (Table 4). Vitreoretinal interface abnormality was the third cause of monocular mild visual impairment and MSVI, accounting for $2.2 \%$ and $4.4 \%$ of all cases, respectively. The leading causes of monocular blindness were cataract (32.7\%), corneal opacity (12.7\%), and atrophy of the eyeball or prosthetic eye $(9.7 \%)$.

Altogether, a large number of bilateral and monocular visual impairment cases $(81.7 \%$ and $77.1 \%$, respectively) were caused by treatable pathologies including cataract, uncorrected refractive error, and posterior capsular opacification.

\section{Factors Associated with Visual Impairment}

Bilateral MSVI and blindness were associated with age (both $p<0.001)$ and sex $(p<0.001$ and $p=0.018$, respectively) 
Table I Characteristics for Participants and Nonparticipants

\begin{tabular}{|l|c|c|c|c|}
\hline & Participants (n=1914), \% & Nonparticipants (n=250), \% & Response Rate & value* \\
\hline Age (yrs, Mean \pm SD) & $79.1 \pm 4.6$ & $80.1 \pm 4.9$ & $91.7 \%$ & 0.002 \\
$70-74$ & 16.1 & 11.2 & $89.7 \%$ & 0.014 \\
$75-79$ & 40.6 & 35.6 & $86.9 \%$ & \\
$80-84$ & 29.4 & 34.0 & $84.8 \%$ & \\
$85 \sim$ & 13.9 & 19.2 & & \\
\hline Gender & & & $89.2 \%$ & \\
Male & 46.0 & 42.4 & $87.8 \%$ & \\
Female & 54.0 & 57.6 & & \\
\hline Education & & & $88.0 \%$ & \\
Less than primary & 46.9 & 50.6 & $88.7 \%$ & \\
Primary & 29.5 & 29.9 & $90.6 \%$ & \\
Secondary or higher & 23.6 & 19.5 & $88.4 \%$ & \\
Total & 100.0 & 100.0 & \\
\hline
\end{tabular}

Note: *Student's $t$-test for continuous variables, and chi-square for categorical variables. Abbreviation: SD, standard deviation.

(Figure 2). Table 5 shows the univariate and multivariate logistic regression results of the factors associated with visual impairment. Advanced age (per year, odds ratio [OR], 1.09; 95\% CI, 1.06-1.12; $p<0.001$ ), female sex (OR, 1.59; 95\% CI, $1.18-2.15 ; p=0.003)$, self-reported visual impairment (OR, $1.91 ; 95 \% \mathrm{CI}, 1.51-2.41 ; p<0.001)$, and cognitive impairment (OR, 1.40; 95\% CI, 1.11-1.76, $p=0.005$ ) were significantly associated with a higher prevalence of visual impairment. By contrast, higher educational level (OR, 0.75 ; $95 \%$ CI, $0.56-0.99 ; \mathrm{p}=0.045$ for primary; OR, 0.53 ; $95 \%$ CI, $0.38-0.74 ; p<0.001$ for secondary or higher) was significantly associated with a lower prevalence of visual impairment.

\section{Discussion}

The current study is a large population-based survey that is aimed to assess the current prevalence and causes of visual impairment in an elderly Chinese population aged $\geq 70$ years. After adjusting the China National Census for age and gender, $48.9 \%$ of the elderly individuals had bilateral visual impairment, of whom $20.5 \%$ had mild visual impairment, 25.8\% had bilateral MSVI, and 3.4\% were blind, according to the WHO criteria. In mainland China, approximately 38.1 million elderly individuals aged $\geq 70$ years have visual impairment, of whom 19.5 million had MSVI and 2.6 million were blind. China is experiencing a rapid population aging; the population of adults aged $\geq 70$ years is projected to reach 290 million by $2050 .^{22}$ This trend of societal aging may result in 140 million elderly people aged $\geq 70$ years developing bilateral visual impairment in China by 2050. Cataract and uncorrected refractive errors are the leading causes of visual impairment. In particular, a high prevalence of vitreoretinal interface abnormality-related visual impairment was detected by OCT and fundus examination.

The prevalence of visual impairment varies among different regions in China and in other countries, based on the WHO criteria for PVA and BCVA in the better eye (Supplementary Table 2 and Supplementary Table 3). In the current study, the prevalence of MSVI and blindness among adults aged $\geq 70$ years was comparable to that in the Nine Provinces Eye Survey ${ }^{23}$ and Taizhou study. ${ }^{10}$ On the contrary, a remarkably lower prevalence of best-corrected visual impairment among the elderly population was observed in the Shihpai Eye Study that included elderly people aged $\geq 65$ years $(8.2 \%$ vs $33.1 \%$ in the current study). This discrepancy was due to the significantly higher prevalence of visual impairment in rural areas than in urban areas. ${ }^{7,8}$ The lack of ophthalmologists, limited facilities, and transportation barriers between rural and urban regions are significant obstacles that need to be addressed during the expansion of eye care services to rural residents in China.

Compared with other countries, the prevalence of visual impairment in mainland China was higher than that observed in developed countries (Supplementary Table 2 and Supplementary Table 3). These substantial differences could be explained by the variations in 


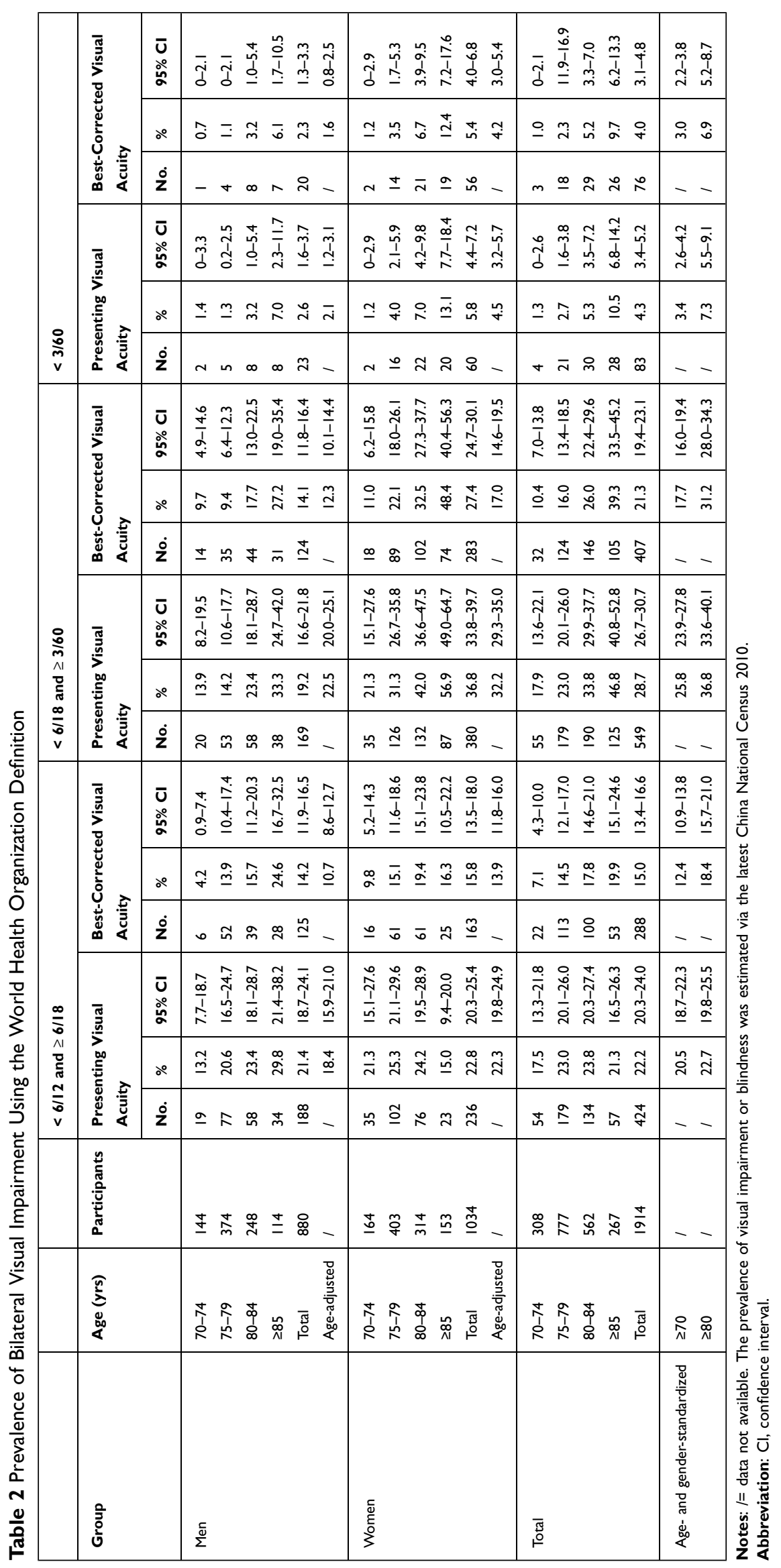




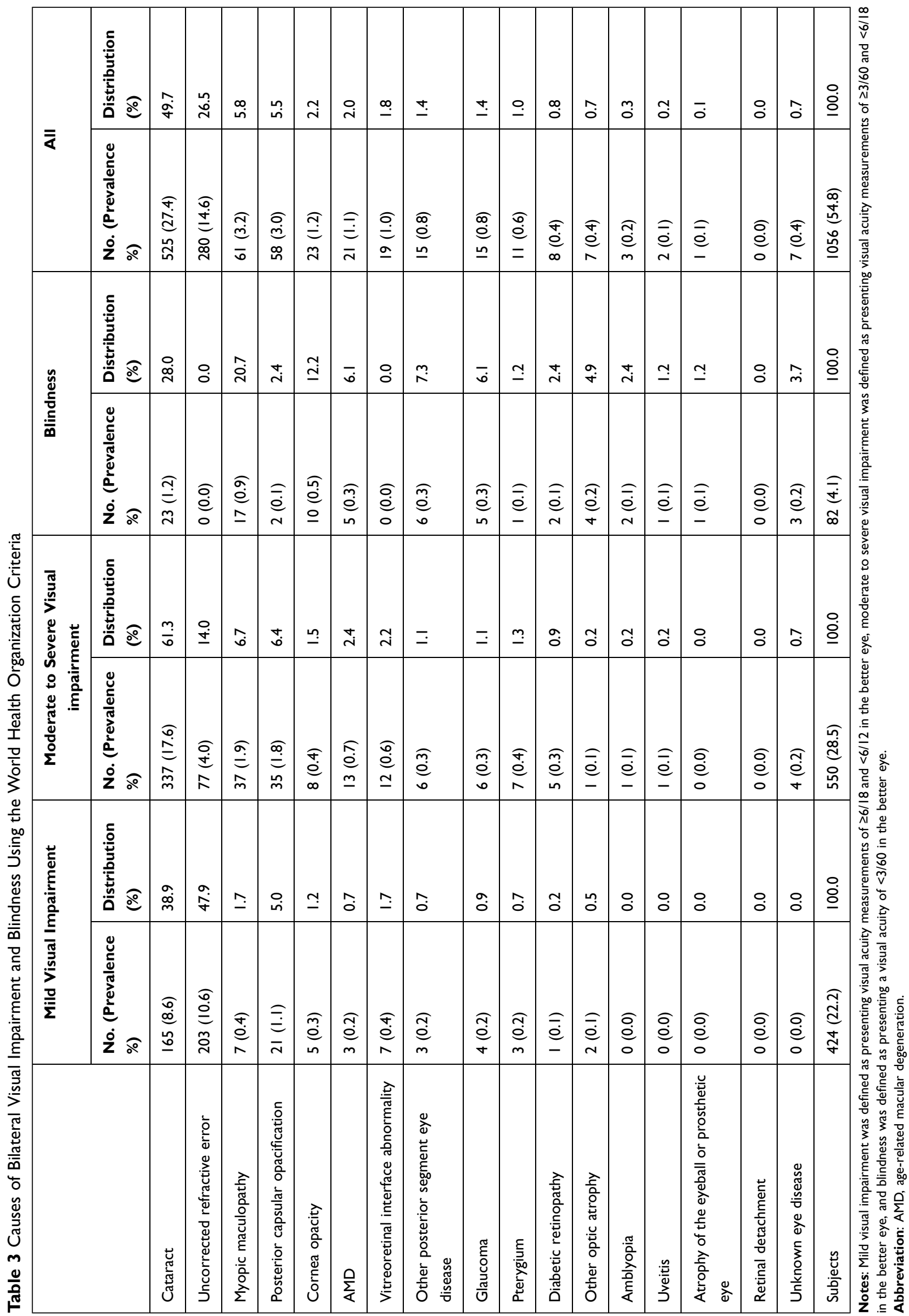




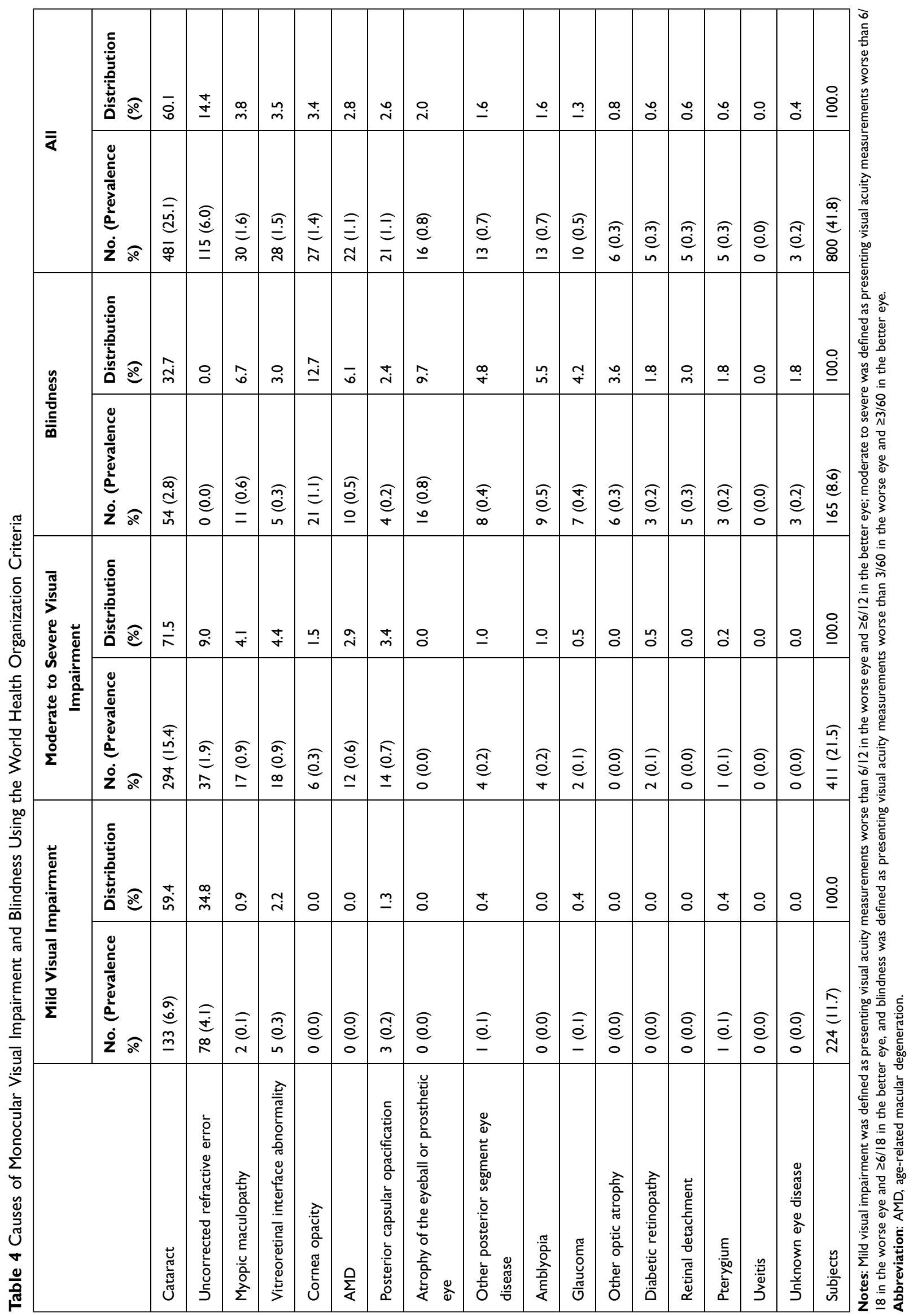



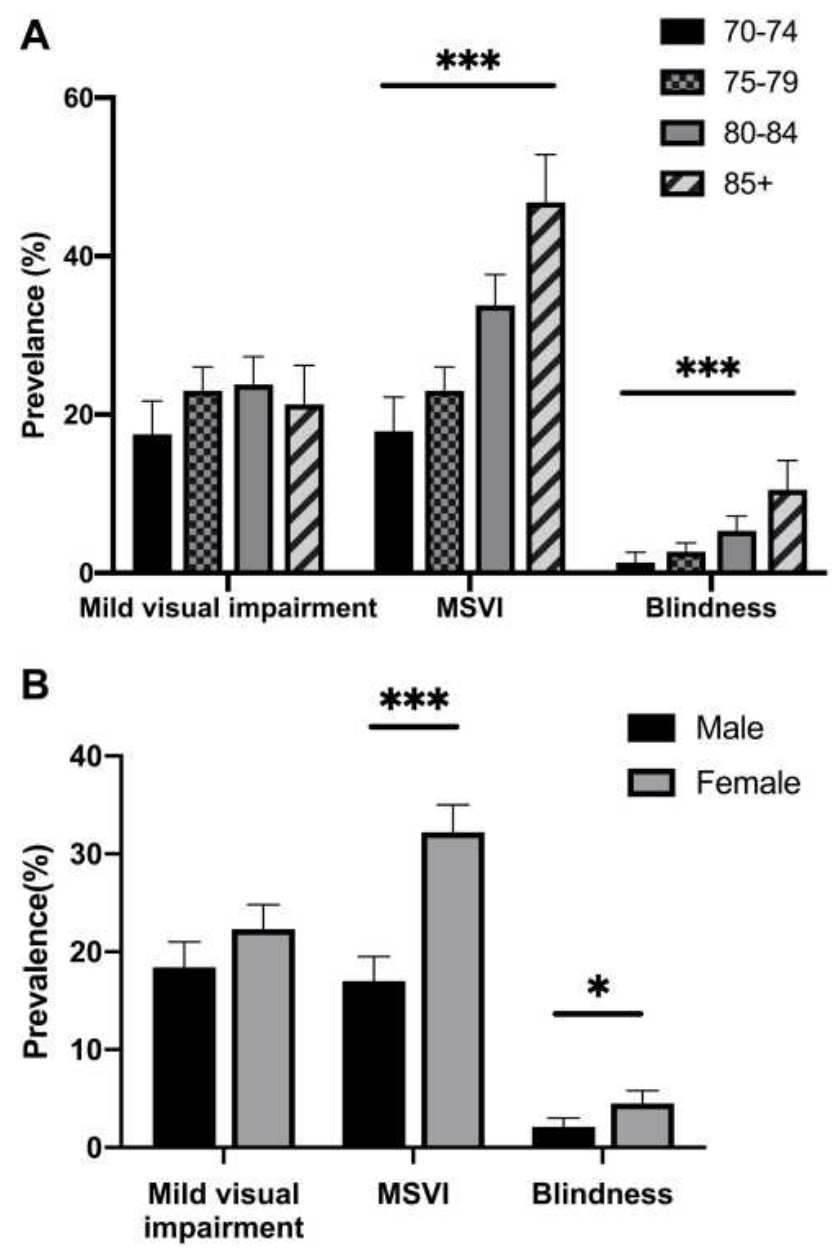

Figure 2 The prevalence of presenting visual impairment using the World Health Organization standard. (A) The prevalence of MSVI and blindness increased with age. (B) The prevalence of MSVI and blindness was higher in female than those in male. ${ }^{*} p<0.05 ; * * * p<0.001$.

Abbreviation: MSVI, moderate to severe visual impairment.

definitions, demographic makeup, socioeconomic level, sampling frame, and study time. In addition, the elderly population in China might be less likely to seek eye healthcare. The Chinese Health Statistical Yearbook 2019 reported that there were over 40,000 registered ophthalmologists in China, more than twice as many as in $2010 .{ }^{24}$ However, in a study by Peng et $\mathrm{al}^{25}$ nearly $90 \%$ of the Chinese rural population had never sought eye care services, for whom education to raise the awareness of eye diseases is needed to reduce visual impairment.

Cataract and uncorrected refractive errors were the main causes of visual impairment in the elderly population. This finding is similar to that of other populationbased studies. ${ }^{10,23}$ A novel finding in our study was that posterior capsular opacification was the fourth leading cause of visual impairment. This could be explained by the increasing cataract surgery rate promoted by the
Chinese government over decades. ${ }^{26}$ Based on our study findings, a large proportion (81.7\%) of visual impairment can be significantly improved by cataract surgery, refractive correction, or Nd:YAG laser capsulotomy. These results suggest that the prevention of visual impairment caused by cataract is well under way; however, further efforts are needed for the government and hospitals to increase cataract surgery coverage and postoperative follow-up, as well as provide spectacle-dispensing services for elderly people living in the rural areas in China.

Myopic maculopathy was the leading cause of retinal diseases, which is consistent with the findings in other eye studies in Asia. ${ }^{27}$ The prevalence of myopic maculopathy-related visual impairment in Asians is higher than that in the Western population, ${ }^{28}$ which requires further investigation.

We observed a high prevalence of vitreoretinal interface abnormalities leading to bilateral and monocular mild visual impairment $(1.7 \%$ and $2.2 \%$, respectively) and MSVI (2.2\% and $4.4 \%$, respectively), using OCT scan. OCT provides more sensitive and specific assessments of the vitreoretinal interface, compared with fundus photography. ${ }^{29}$ However, the examination of the vitreoretinal interface in most population-based studies was limited to fundus photography. The prevalence of visual-impairing vitreoretinal interface abnormalities in our study $(1.0 \%)$ was higher than that in the Hong Kong study $(0.05 \%),{ }^{30}$ but lower than that in the UK Biobank Study $(3.6 \%) .{ }^{31}$ As the incidence of vitreoretinal interface abnormalities increase with age, ${ }^{32}$ future population-based studies must include this as a potential cause of visual impairment among the elderly population.

The higher prevalence of visual impairment was associated with advanced age, female sex, self-reported visual impairment, and cognitive impairment, which is consistent with the results of previous studies. ${ }^{8,28}$ Individuals with good cognitive function are more likely to seek eye care, while good vision helps mitigate age-related cognitive decline. ${ }^{33}$ A higher educational level was associated with a lower risk of visual impairment. These findings may help to effectively identify interventional strategies to combat visual impairment.

The present study had several strengths. First, the large and representative study sample in this elderly population with a high response rate allowed us to obtain credible findings. Population-based studies in the elderly are usually limited by a high frequency of physical and cognitive disability. Our sample size $(\mathrm{n}=1914$; response rate, $88.4 \%)$ was 
Table 5 Logistic Regression Analysis for Factors Associated with Bilateral Visual Impairment (Presenting Visual Acuity < 6/I2 in the Better Eye)

\begin{tabular}{|c|c|c|c|c|}
\hline Variables & Univariate Odds Ratio $(95 \% \mathrm{Cl})^{\mathrm{a}}$ & $p$ value & Multivariate Odds Ratio $(95 \% \mathrm{Cl})^{\mathrm{b}}$ & $p$ value \\
\hline Age (per year) & $1.13(1.11-1.16)$ & $<0.001$ & $1.09(1.06-1.12)$ & $<0.001$ \\
\hline Female gender & $2.49(2.07-2.99)$ & $<0.001$ & $1.59(1.18-2.15)$ & 0.003 \\
\hline Married & $0.62(0.51-0.76)$ & $<0.001$ & $1.14(0.90-1.44)$ & 0.30 \\
\hline \multicolumn{5}{|l|}{ Education } \\
\hline Less than primary & Reference & & Reference & \\
\hline Primary & $0.40(0.32-0.50)$ & $<0.001$ & $0.75(0.56-0.99)$ & 0.045 \\
\hline Secondary or higher & $0.26(0.2 \mathrm{I}-0.34)$ & $<0.001$ & $0.53(0.38-0.74)$ & $<0.001$ \\
\hline History of smoking & $0.59(0.47-0.73)$ & $<0.001$ & I.I2 (0.83-I.5I) & 0.45 \\
\hline History of alcohol & $0.60(0.49-0.73)$ & $<0.001$ & $0.84(0.65-1.07)$ & 0.15 \\
\hline Self-reported visual impairment & $2.17(1.77-2.67)$ & $<0.001$ & $1.91(I .5 I-2.4 I)$ & $<0.001$ \\
\hline \multicolumn{5}{|l|}{ BMI $\left(\mathrm{kg} / \mathrm{m}^{2}\right)$} \\
\hline Normal (I8.0-23.9) & Reference & & Reference & \\
\hline Underweight $(<18.0)$ & $1.55(0.95-2.54)$ & 0.24 & $0.88(0.48-1.6 \mathrm{I})$ & 0.67 \\
\hline Overweight (24.0-27.9) & $0.79(0.65-0.97)$ & $<0.001$ & $0.79(0.63-1.01)$ & 0.059 \\
\hline Obese $(\geq 28.0)$ & $0.77(0.57-1.05)$ & 0.85 & $0.83(0.57-1.19)$ & 0.31 \\
\hline Systolic blood pressure $(\mathrm{mmHg})$ & $1.00(1.00-1.00)$ & 0.87 & & \\
\hline Diastolic blood pressure $(\mathrm{mmHg})$ & $1.00(0.99-1.00)$ & 0.16 & & \\
\hline Fasting blood glucose (mmol/L) & $1.00(0.95-1.05)$ & 0.92 & & \\
\hline Serum-HbAlc (\%) & $0.95(0.86-1.04)$ & 0.27 & & \\
\hline Serum-Homocysteine (umol/L) & $1.01(1.00-1.02)$ & 0.07 & & \\
\hline Serum-cholesterol (mmol/L) & I.0I $(0.93-1.1 \mathrm{I})$ & 0.78 & & \\
\hline Cognitive impairment ${ }^{c}$ & $2.24(1.82-2.74)$ & $<0.001$ & $1.40(1.11-1.76)$ & 0.005 \\
\hline
\end{tabular}

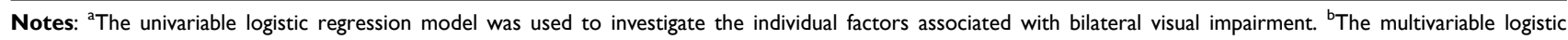
regression, including factors with association in the univariable analysis with a $p$ value of $<0.05$, was used to investigate the factors associated with bilateral visual impairment. ${ }^{c}$ Cognitive impairment was defined as Mini-Mental State Examination scores of $\leq 16$ for illiterate, $\leq 19$ for individuals with $1-6$ years of education, and $\leq 23$ for individuals with 7 or more years of education.

Abbreviations: $\mathrm{Cl}$, confidence interval; HbAlc, glycated hemoglobin; BMI, body mass index.

higher than those in other studies of this age group ${ }^{17}$ and was well matched in terms of national gender ratio (female ratio in the age group of 70 years and older, 53.1\% in national census versus $54.0 \%$ in the current study), which minimized the selection bias. Second, the use of standardized protocols as well as comprehensive ophthalmic examinations, including OCT scans, improved the accuracy of evaluations of visual impairment prevalence and causes.

This study has several limitations that should be addressed. First, the non-participants were older than the participants. As the rates of vision loss increase significantly with age, this might result in an underestimation of the true prevalence of visual impairment. Second, as cataract accounted for most cases of visual impairment, concurrent retinal diseases and glaucoma might have been masked by severe lens opacity among the elderly individuals. Third, the examinations were carried out without cycloplegia, which may have contributed to the failure to detect some conditions. Fourth, definitions of visual impairment were limited to distance visual acuity, while near visual impairment and visual field were not assessed, which may have underestimated the prevalence of uncorrected refractive error, glaucoma or other optic atrophy. In addition, Given the cross-sectional nature of the study, our findings only suggested the associations between visual impairment and factors, but not a causative relationship.

\section{Conclusion}

In conclusion, RuLAS presented a high prevalence of visual impairment in an elderly Chinese population. Cataract and uncorrected refractive error remain the leading causes of vision loss, highlighting the significant challenges in popularizing cataract surgery and the use of spectacles for the elderly individuals living in rural areas. Myopic maculopathy, vitreoretinal interface abnormality, and posterior capsular opacification also contributed significantly to visual impairment. Strengthening the awareness of eye health 
care should be listed as a high priority in health care services in rural areas in China, and specific focus should be placed on the eye health status of elderly women with low educational levels and cognitive impairment.

\section{Abbreviations}

AMD, age-related macular degeneration; BCVA, bestcorrected visual acuity; BMI, body mass index; CI, confidence interval; MMSE, Mini-Mental State Examination; MSVI, moderate to severe visual impairment; OCT, optical coherence tomography; OR, odds ratio; PVA, presenting visual acuity; RuLAS, Rugao Longevity and Aging Study; WHO, World Health Organization.

\section{Ethics Approval and Informed Consent}

Obtained.

\section{Acknowledgment}

The authors thank Dr. Wenrui Ma, MD, Shanghai Fudan University, for editing the manuscript.

\section{Funding}

This work was financially supported by grants from the National Natural Science Foundation of China (81970810, 81500714, 81670465, 81600577, 81800835, 81970811, $82002891)$, the National Key R\&D Program of China (2016YFC0904800, 2018YFC2000400, 2018YFC2002000, 2019YFC0840607), and National Science and Technology Major Project of China (2017ZX09304010).

\section{Disclosure}

The following authors have no proprietary or commercial interest in any materials discussed in this article: Hangqi Shen, Hui Zhang, Wei Gong, Tianwei Qian, Tianyu Cheng, Li Jin, Xiaofeng Wang, Dawei Luo, and Xun Xu.

\section{References}

1. World Health Organization. World report on vision. Geneva: World Health Organization; 2019. Available from: https://www.who.int/pub lications/i/item/world-report-on-vision. Accessed October 8, 2019

2. Vu HT, Keeffe JE, McCarty CA, Taylor HR. Impact of unilateral and bilateral vision loss on quality of life. Br J Ophthalmol. 2005;89 (3):360-363. doi:10.1136/bjo.2004.047498

3. Liu Z, Wu D, Huang J, et al. Visual impairment, but not hearing impairment, is independently associated with lower subjective well-being among individuals over 95 years of age: a population-based study. Arch Gerontol Geriatr. 2016;62:30-35. doi:10.1016/j.archger.2015.10.011
4. Heesterbeek TJ, Van der Aa HPA, Van Rens G, Twisk JWR, Van Nispen RMA. The incidence and predictors of depressive and anxiety symptoms in older adults with vision impairment: a longitudinal prospective cohort study. Ophthalmic Physiol Opt. 2017;37 (4):385-398. doi:10.1111/opo.12388

5. United Nations. Population Division. World population ageing, 2019: highlights; 2019. Available from: https://digitallibrary.un.org/record/ 3846855. Accessed January 14, 2020.

6. Fang EF, Scheibye-Knudsen M, Jahn HJ, et al. A research agenda for aging in China in the 21st century. Ageing Res Rev. 2015;24:197-205. doi:10.1016/j.arr.2015.08.003

7. Xu L, Wang Y, Li Y, et al. Causes of blindness and visual impairment in urban and rural areas in Beijing: the Beijing Eye Study. Ophthalmology. 2006;113(7):1134 e1-11. doi:10.1016/j. ophtha.2006.01.035

8. Liang YB, Friedman DS, Wong TY, et al. Prevalence and causes of low vision and blindness in a rural chinese adult population: the Handan Eye Study. Ophthalmology. 2008;115(11):1965-1972. doi:10.1016/j.ophtha.2008.05.030

9. Huang S, Zheng Y, Foster PJ, Huang W, He M; Liwan Eye Study. Prevalence and causes of visual impairment in Chinese adults in urban southern China. Arch Ophthalmol. 2009;127(10):1362-1367. doi:10.1001/archophthalmol.2009.138

10. Tang Y, Wang X, Wang J, et al. Prevalence and causes of visual impairment in a Chinese adult population: the Taizhou Eye Study. Ophthalmology. 2015;122(7):1480-1488. doi:10.1016/j. ophtha.2015.03.022

11. Liu Z, Wang Y, Zhang Y, et al. Cohort profile: the Rugao Longevity and Ageing Study (RuLAS). Int J Epidemiol. 2016;45(4):1064-1073. doi:10.1093/ije/dyv101

12. Zhou B; Coorperative Meta-Analysis Group Of China Obesity Task Force. Predictive values of body mass index and waist circumference to risk factors of related diseases in Chinese adult population. Zhonghua Liu Xing Bing Xue Za Zhi. 2002;23(1):5-10.

13. Li H, Jia J, Yang Z, Moreau N. Mini-mental state examination in elderly Chinese: a population-based normative study. J Alzheimer's Dis. 2016;53(2):487-496. doi:10.3233/JAD-160119

14. Zhao J, Ellwein LB, Cui H, et al. Prevalence of vision impairment in older adults in rural China: the China Nine-Province Survey. Ophthalmology. 2010;117(3):409-416.e1. doi:10.1016/j. ophtha.2009.11.023

15. Varma R, Vajaranant TS, Burkemper B, et al. Visual impairment and blindness in adults in the United States: demographic and geographic variations from 2015 to 2050. JAMA Ophthalmol. 2016;134 (7):802-809. doi:10.1001/jamaophthalmol.2016.1284

16. World Health Organization. International classification of diseases, Tenth Revision (ICD-10); 2019. Available from: https://icd.who.int/ browse10/2019/en\#/H54. Accessed October 25, 2020.

17. Marmamula S, Barrenakala NR, Challa R, et al. Prevalence and risk factors for visual impairment among elderly residents in 'homes for the aged' in India: the Hyderabad Ocular Morbidity in Elderly Study (HOMES). Br J Ophthalmol. 2021;105(1):32-36. doi:10.1136/ bjophthalmol-2019-315678

18. Chylack LT Jr., Wolfe JK, Singer DM, et al. The lens opacities classification system III. The Longitudinal Study of Cataract Study Group. Arch Ophthalmol. 1993;111(6):831-836. doi:10.1001/ archopht.1993.01090060119035

19. Kuang TM, Tsai SY, Liu CJL, Ko YC, Lee SM, Chou P. Seven-year incidence of uncorrected refractive error among an elderly Chinese population in Shihpai, Taiwan: the Shihpai Eye Study. Eye. 2016;30 (4):570-576. doi:10.1038/eye.2015.276

20. Ohno-Matsui K, Kawasaki R, Jonas JB, et al. International photographic classification and grading system for myopic maculopathy. Am J Ophthalmol. 2015;159(5):877-883.e7. doi:10.1016/j. ajo.2015.01.022 
21. Duker JS, Kaiser PK, Binder S, et al. The International Vitreomacular Traction Study Group Classification of vitreomacular adhesion, traction, and macular hole. Ophthalmology. 2013;120(12):2611-2619. doi:10.1016/j.ophtha.2013.07.042

22. Chen Y, Liu Z, Huang Y, et al. The aging trend of Chinese population and the prediction of aging population in 2015-2050 [in Chinese]; 2018. http://d.wanfangdata.com.cn/periodical/gwyx-shyx201805013. Accessed October, 2018.

23. Zhao J, Xu X, Ellwein LB, et al. Prevalence of vision impairment in older adults in rural China in 2014 and comparisons with the 2006 China Nine-Province Survey. Am J Ophthalmol. 2018;185:81-93. doi:10.1016/j.ajo.2017.10.016

24. National Health Commission of the People's Republic of China. Chinese health statistical yearbook 2019. Available from: http:// www.nhc.gov.cn/zwgk/tjnj1/ejlist.shtml. Accessed October 25, 2020.

25. Peng Y, Tao QS, Liang YB, et al. Eye care use among rural adults in China: the Handan Eye Study. Ophthalmic Epidemiol. 2013;20 (5):274-280. doi:10.3109/09286586.2013.823216

26. Wong IYH, Ni MY, Wong IOL, Fong N, Leung GM. Saving sight in China and beyond: the Lifeline Express model. BMJ Glob Health. 2018;3(4):e000766. doi:10.1136/bmjgh-2018-000766

27. Iwase A, Araie M, Tomidokoro A, et al. Prevalence and causes of low vision and blindness in a Japanese adult population: the Tajimi Study. Ophthalmology. 2006;113(8):1354-1362. doi:10.1016/j. ophtha.2006.04.022

28. Varma R, Ying-Lai M, Klein R, Azen SP; Los Angeles Latino Eye Study Group. Prevalence and risk indicators of visual impairment and blindness in Latinos: the Los Angeles Latino Eye Study. Ophthalmology. 2004;111(6):1132-1140. doi:10.1016/j.ophtha. 2004.02.002
29. Meuer SM, Myers CE, Klein BEK, et al. The epidemiology of vitreoretinal interface abnormalities as detected by spectral-domain optical coherence tomography. Ophthalmology. 2015;122 (4):787-795. doi:10.1016/j.ophtha.2014.10.014

30. You QS, Choy BKN, Chan JCH, et al. Prevalence and causes of visual impairment and blindness among adult Chinese in Hong Kong-The Hong Kong Eye Study. Ophthalmic Epidemiol. 2020;27 (5):354-363. doi:10.1080/09286586.2020.1755444

31. McKibbin M, Farragher T, Shickle D. Vitreoretinal interface abnormalities in middle-aged adults with visual impairment in the UK Biobank study: prevalence, impact on visual acuity and associations. BMJ Open Ophthalmol. 2017;1(1):e000057. doi:10.1136/bmjophth-2016-000057

32. Mitchell P, Smith W, Chey T, Wang JJ, Chang A. Prevalence and associations of epiretinal membranes. The Blue Mountains Eye Study, Australia. Ophthalmology. 1997;104(6):1033-1040. doi:10.1016/S0161-6420(97)30190-0

33. Zheng DD, Swenor BK, Christ SL, West SK, Lam BL, Lee DJ. Longitudinal Associations Between Visual Impairment and Cognitive Functioning: the Salisbury Eye Evaluation Study. JAMA Ophthalmol. 2018;136(9):989-995. doi:10.1001/jamaophthalmol. 2018.2493
Clinical Interventions in Aging

\section{Publish your work in this journal}

Clinical Interventions in Aging is an international, peer-reviewed journal focusing on evidence-based reports on the value or lack thereof of treatments intended to prevent or delay the onset of maladaptive correlates of aging in human beings. This journal is indexed on PubMed Central, MedLine, CAS, Scopus and the Elsevier

\section{Dovepress}

Bibliographic databases. The manuscript management system is completely online and includes a very quick and fair peer-review system, which is all easy to use. Visit http://www.dovepress.com/ testimonials.php to read real quotes from published authors. 